\section{Should Therapeutic Monitoring of Vancomycin Based on Area under the Curve Become Standard Practice for Patients with Confirmed or Suspected Methicillin-Resistant Staphylococcus aureus Infection? The "Pro" Side: Correction}

Original citation: Claeys KC, Brade KD, Heil EL. Should therapeutic monitoring of vancomycin based on area under the curve become standard practice for patients with confirmed or suspected methicillin-resistant Staphylococcus aureus infection? The "Pro" side. Can J Hosp Pharm. 2020;73(3):232-4.
In the Point Counterpoint debate in the May-June 2020 issue, paragraph 4 of the "Pro" article contained some errors. Specifically, reference 11 should not have been cited with the opening sentence, and that sentence should have ended with the word "limited" (not "lacking"); the complete sentence should read as follows: "Data correlating attainment of the target vancomycin trough with improved clinical outcomes are limited." In the same paragraph, the fourth and fifth sentences should be replaced with the following corrected sentence: "Kullar and others" ${ }^{11}$ [Clin Infect Dis. 2011;52(8):975-81] found an association between trough and clinical outcomes and also found a similar result with respect to the AUC/MIC cut-off as above." 